\title{
Vies et morts de l'imagination : La puissance des actes fantômes
}

Par ANNABELLE DUfOURCQ

Radboud University, Nijmegen

Résumé Imaginer consiste-t-il en un acte ou en une catastrophe ontologique, l'inévitable dissolution de l'être, l'expérience de la mort au cœur de la vie? Partant des deux versions de l'imaginaire définies par Blanchot, je propose avec Husserl une troisième voie, celle de l'imagination comme dialogue avec des actes fantômes. Il y a bien chez Husserl une théorie des actes d'imagination, mais également la mise en place d'une conceptualité permettant de penser la dimension ontologique de l'imaginaire : la phénoménalité de la chose même implique le pouvoir de paraître en image, à la fois ici et là, présente et absente, flottante. Et cette modalité d'être est cruciale dans le cadre d'une philosophie dont l'un des thèmes clefs est celui de la mort et de la résurrection du sens. Toutefois dans l'approche phénoménologique husserlienne il n'y a pas de mort absolue et c'est bien plutôt une ontologie des fantômes qui voit le jour.

\section{Introduction}

Cet article trouve son origine dans une réflexion inspirée par le thème et l'argumentaire du présent colloque. C'est cette notion d'acte qui est au cœur de mon questionnement. Qu'est-ce au juste que l'acte d'imagination? Mais, avant cela : faut-il penser l'imagination d'abord comme un acte?

Que se passe-t-il au juste dans l'image ou lorsque j'imagine? Blanchot définit deux voies possibles, deux modes de l'image dans L'espace littéraire («Les deux versions de l'imaginaire»). D'abord la face rayonnante et heureuse de l'imaginaire : l'image redonne vie à l'objet disparu et toutes les puissances de distanciation et de déréalisation sont mises au service d'un 
procès d'idéalisation. «Le reflet ne paraît-il pas toujours plus spirituel que l'objet reflété ? N'est-il pas de cet objet l'expression idéale, la présence libérée de l'existence, la forme sans matière ? ${ }^{1}$. Il y a déjà abstraction dans et par l'image d'un corps glorieux et d'un esprit traversant les incarnations. Mais un discret écœurement pointe déjà, une tragédie également. Apparaît ici la deuxième version de l'imaginaire. La dissolution, l'effondrement qui étaient à l'œuvre en arrière-plan et sous une forme très contenue dans cette première version apollinienne, excèdent en fait cette forme et, si le sujet imaginant peut avoir l'impression de jouer avec eux, d'en faire usage, il devra aussi pressentir que ce qui se profile le transcende infiniment : c'est l'insaisissable, le nulle part. C'est aussi le «rien $»^{2}$, le néant. Où est le roi dont je contemple le portrait? Ici et ailleurs, ni ici ni ailleurs vraiment, descellé, disloqué, en porte-à-faux, nulle part vraiment. L'objet dans son image apparaît sur fond d'un processus de perte de soi, perte d'ancrage, d'identité, de substance et d'être tel que, même si cet objet dans l'image est bien, certes, encore là, il se dévoile comme n'étant pas de droit toujours pleinement là, pleinement lui-même, et donc surgi du non-sens.

Les deux versions de l'imaginaire sont ainsi les suivantes : l'éloignement au service du mieux voir et du mieux connaître la chose ou « l'éloignement au cœur de la chose $»^{3}$, la chose dévorée par l'éloignement, rongée par son image. L'image nous dit que la chose peut disparaître, n'être plus que quasiment là, donc, qu'elle a toujours été minée, qu'elle n'a jamais été vraiment complètement là. S'ensuit une analyse de l'image sur le modèle du cadavre : le cadavre est ici, pesamment présent en ce lieu, mais pour nous dire l'horreur de l'absence « image insoutenable et figure de l'unique devenant n'importe quoi $»^{4}:$ «Le cadavre n'est pas à sa place. Où est-il ? Il n'est pas ici et pourtant il n'est pas ailleurs ; nulle part ? C'est qu'alors nulle part est ici $»^{5}$.

Ce que ces analyses de Blanchot mettent admirablement en évidence c'est que l'image ne suit pas 1 'objet ${ }^{6}$, mais renvoie à une négativité ontologique qui précède l'objet et qui rend également possibles les actes d'imagination. $\mathrm{Si}$, dans la première version de l'imaginaire, il est encore question de l'acte d'imaginer - le sujet s'approprie l'écart, l'irréalisation, pour mieux penser

1 M. Blanchot, L'espace Littéraire, Paris, Gallimard, 1955, p. 268.

2 Ibid., p. 271.

3 Ibid., p. 268.

4 Ibid., p. 270.

5 Ibid., p. 269.

6 Ibid., p. 133. 
le monde : nous tenons à distance des choses pour en disposer - , dans la seconde version « la distance nous tient ${ }^{1}$. " L'image est [alors] le moment de la passivité $»^{2}$, c'est la «pure passion de l'indifférence $»^{3}$ qui l'emporte : non plus «négation vivifiante» mais «pesanteur» ${ }^{4}$ et chute abyssale vers l'indéterminé.

On trouve ici véritablement l'antithèse de la notion d'acte d'imagination. L'antithèse radicale, trop peut-être. J'ai repris l'introduction par Blanchot de concepts tels que ceux de néant, de rien, de nulle part, de destruction, de pure passion (passivité) et de mort. Cette grille conceptuelle de lecture de l'imaginaire me semble en fait fortement problématique : je ne suis pas sûre qu'elle soit la plus adéquate pour décrire l'imagination et l'imaginaire. $\mathrm{Ne}$ serait-ce que, comme Blanchot lui-même l'écrit à la fin de ce chapitre, parce que chaque version de l'imaginaire est l'envers de l'autre et porte l'autre à son envers ou son horizon (l'ambiguïté dit Blanchot et non l'une ou l'autre $\left.{ }^{5}\right)$ : de sorte qu'il n'y aurait pas de pure passion et que les morts sont aussi vivants.

C'est précisément sur ce point qu'une approche phénoménologique peut être particulièrement éclairante.

On trouve dans le champ phénoménologique un travail riche et profond sur l'ontologie de l'imaginaire: chez Sartre évidemment, MerleauPonty, Fink, Richir, mais aussi, je vais essayer de le montrer, chez Husserl. Dans le présent article, je souhaite ainsi d'abord démontrer qu'une réflexion phénoménologique sur l'acte d'imagination conduit nécessairement à l'élaboration d'une réflexion sur l'être d'une phénoménalité qui se donne aussi en image, autrement dit, comme le soulignera Blanchot, qui meurt, s'étiole, et, aussi, ressuscite sans cesse.

D'autre part, le second apport crucial de la phénoménologie à cette question de la vie et de la mort des choses en image tient, me semble-t-il, dans le dépassement de la radicale opposition entre vie et mort. Très vite pour Husserl le problème de l'imagination est celui du mélange entre présence et absence, de la presque-mort du sens et de sa toujours possible résurrection, de l'éternel retour des morts, plus exactement et par conséquent : des fantômes.

1 Ibid., p. 274.

2 Ibid., p. 275.

3 Ibid.

4 Ibid.

5 Ibid., p. 276. 
Mon propos sera donc de montrer que la réflexion phénoménologique sur l'acte d'imagination, particulièrement déjà chez Husserl, conduit à une ontologie qui atténue la portée de ce terme d'acte, mais également, d'autre part, à une ontologie des fantômes (une hantologie avant l'heure, comme il se doit). Il ne s'agit pas tellement de rompre avec le concept d'acte d'imagination mais plutôt de le penser en dialogue essentiel avec un imaginaire ontologique et une imagination anonyme.

Comment passe-t-on d'une phénoménologie de l'imagination, d'une description phénoménologique des images et des actes imaginants à une ontologie de l'imagination dans les choses et qu'est-ce que cela signifie exactement? Pourquoi une ontologie des fantômes plutôt que du néant ? Je commencerai par étudier la description phénoménologique de la présence/absence des êtres dans l'imagination. Puis je montrerai que la pensée husserlienne de l'imagination recèle une tension entre une conception en termes d'actes, disons transcendantale, centrée sur la présence et une conception qui découvre le soubassement de passivité de toute imagination et nous met sur la voie d'une ontologie de l'imaginaire. Enfin, dans la dernière partie de cet article, j'expliquerai pourquoi, selon moi, il faut concevoir la dimension ontologique fantomatique de toute chose, condition de possibilité de nos actes d'imagination, comme un ensemble d'actes fantômes et une imagination anonyme, plutôt que comme une indétermination ou une ambiguité en soi ou comme un néant abyssal.

\section{Description phénoménologique de la présence/absence des êtres dans l'imagination}

C'est désormais un pan de la philosophie de Husserl qui est bien connu. J'insisterai de façon synthétique, sur deux points: avec l'imagination il s'agit de penser une certaine forme de présence de la chose imaginée, mais également, d'autre part, une présence fluctuante.

Pour commencer, l'originalité fondamentale de la pensée husserlienne de l'imagination tient dans la thèse selon laquelle l'imagination est une intuition, thèse clairement avancée dans les Recherches logiques ${ }^{1}$ puis reprise de façon constante dans les œuvres suivantes. Imaginer, selon

1 E. Husserl, Logische Untersuchungen, Zweiter Teil. Untersuchungen zur Phänomenologie und Theorie der Erkenntnis, en deux tomes, La Haye, Martinus Nijhoff, 1984, trad. fr. H. Elie, A. L. Kelkel et R. Schérer, Recherches logiques, Paris, P.U.F., 1959-1963, $5^{\mathrm{e}}$ Recherche, $\S 44$ et $6^{\mathrm{e}}$ Recherche, $\S 14$. 
Husserl, n'est pas essentiellement viser un objet inexistant ou absent, ni seulement faire comme s'il était là, c'est vivre une expérience de quasiprésence qui atteint des formes culminantes lorsque, par exemple, j'éprouve, malgré moi, que le personnage dans le portrait me regarde ${ }^{1}$.

D'autre part, Husserl entend également penser bien évidemment la différence entre imagination et perception, mais aussi une ample échelle de variations au sein même du registre de l'imaginaire. Celle-ci conduit de la quasi-présence fascinante, il faudrait même parler d'hyper-présence dans un certain nombre de $\operatorname{cas}^{2}$, jusqu'à l'image abstraite, les symboles, hiéroglyphes et schémas manipulés par une pensée plus objectivante afin de re-présenter, à distance, l'objet-visé. Dans ce second cas, l'image apparaît plus clairement comme la cousine du signe (lequel est défini par Husserl comme absence d'intuition, absence de remplissement, visée à vide). Ainsi dans le cours de 1904-1905, premier texte du $23^{\mathrm{e}}$ tome des Husserliana, Husserl met à plusieurs reprises en avant la continuité entre signes et images : il place l'origine des signes dans les symboles, c'est-à-dire fondamentalement dans une relation de ressemblance avec l'objet désigné ${ }^{3}$, et ajoute que, inversement, toute image tend à se dégrader en signe - c'est-à-dire en un renvoi à un objet qui lui est extérieur. L'image n'est pas présence de la chose-même et cette frustration finit par l'emporter, devient désir d'autres images ou d'une perception $^{4} ; 1$ 'on retrouve ainsi en elle le vide du signe. Reste que si la visée est vide, nous ne sommes plus en présence d'une phantasia ou d'une image : l'imagination consiste donc essentiellement dans la quasi-présence et, d'autre part, une quasi-présence susceptible de s'intensifier jusqu'à l'hyper-présence

1 E. Husserl, Phantasie, Bildbewußtsein, Erinnerung. Zur Phänomenologie der anschaulichen Vergegenwärtigungen, Texte aus dem Nachlaß (1898-1925) (abrégé Hua XXIII), La Haye, Martinus Nijhoff, 1980, p. 30, trad. fr. R. Kassis et J.F. Pestureau, Millon, Grenoble, 2002, p. 72. Sur la notion de quasi-présence je me permets de renvoyer à mon ouvrage La dimension imaginaire du réel dans la philosophie de Husserl, Springer, Phaenomenologica, 2010, particulièrement la première section. 2 Je pense notamment aux cas qui seront particulièrement étudiés par Merleau-Ponty dans L'œil et l'esprit, Paris, Gallimard, 1960 : il arrive que les choses et les personnes acquièrent dans l'image, et notamment dans l'œuvre d'art, une présence plus intense, un rayonnement qui leur manquait dans leur présence perceptive, ainsi la sublimation du banal dans les natures mortes flamandes par exemple.

3 Hua XXIII, § 16.

4 Hua XXIII, $\S 25$, p. $52-53$ (tr. fr. p. 90-91): «Celui qui ne se satisfait pas de l'intuitionner au-dedans peut bien se tourner vers une autre image, meilleure, ou une autre représentation intuitive ». 
et de s'affaiblir au point de négliger ou manquer l'objet au profit de sa représentation.

C'est cette puissance d'intensification/atténuation, d'apparition glorieuse et de disparition qui est visée dans la thématique de vie et mort des images - ou des êtres dans leur image, mais cela va de pair. Les vies endormies (en anglais ou allemand still lives, lebensstile) sont ainsi des natures mortes en français. L'imaginaire collectif nous offre d'innombrables histoires d'images prenant vie, de statues qui saignent ou pleurent, de portraits hantés etc. ${ }^{1}$ De même l'acharnement iconoclaste manifeste toujours, en filigrane, sa contrepartie : une vie retorse des images.

Avant de passer à la manière dont Husserl rend compte de cette quasiprésence et des vies, morts et résurrections des images - ainsi que des choses dans leur image - , je veux souligner que cette dynamique des images confère à l'imagination un rôle charnière spécialement dans la réflexion husserlienne pour laquelle la question de la vie et de la mort des idées est cruciale. En effet, tout d'abord, parce que la phénoménologie étudie les vécus, il lui faut comprendre comment le monde fait sens pour la vie, comme vécu et donc aussi comme vie, puisqu'il n'est pas question de le comprendre comme pur en soi, objet inerte, mais bien comme ce qui est intrinsèquement pour la vie et intrinsèquement le corrélat de la vie. D'autre part cette référence à la vie est cruciale chez Husserl en lien avec le problème de la sédimentation, autrement dit de la minéralisation des idées vivantes. Ce thème est au cœur de la Krisis, mais constitue l'un des motifs originels de la pensée husserlienne. Comment le sens a-t-il pu se perdre, autrement dit comment at-on pu oublier le monde de la vie au profit d'un monde objectif, saisi par l'attitude naturelle comme étranger à toute subjectivité ? Comme le soulignera Baudrillard dans L'échange symbolique et la mort : pour la science il n'est d'objet réel que mort ${ }^{2}$. La Krisis retrace l'histoire d'un positivisme mortifère et présente la phénoménologie comme retour à la vie :

Combattons contre ce danger des dangers [Le naturalisme], (...) et nous verrons alors sortir (...) des cendres de la grande lassitude, le phénix ressuscité d'une nouvelle vie intérieure et d'un nouveau souffle spirituel, gage d'un grand et long avenir pour l'humanité : car l'esprit seul est immortel ${ }^{3}$.

1 Cf. D. Freedberg, The Power of Images, The University of Chicago Press, 1989. 2 J. Baudrillard, L'échange symbolique et la mort, Paris, Gallimard, 1976, p. 205 note.

3 «Kämpfen wir gegen diese Gefahr der Gefahren (...) dann wird (...) aus der Asche der großen Müdigkeit der Phoenix einer neuen Lebensinnerlichkeitdem und 
La philosophie husserlienne est donc essentiellement confrontée au problème de l'intuition comprise comme susceptible de gagner ou perdre la présence, de mourir et de ressusciter. Autrement dit, ce qui constitue l'un des problèmes cruciaux de la phénoménologie husserlienne se joue au cœur du problème de l'imaginaire : une étude de la présence perceptive ne saurait être complètement suffisante et ne pourra pas fournir un paradigme ontologique opérant.

Pourtant il est tentant et c'est une tendance forte chez Husserl, d'exorciser tous ces fantômes en fondant une phénoménologie centrée sur l'intuition comme présence, donation adéquate, ou, en d'autres termes, le modèle cartésien du rapport à soi de l'Ego transcendantal comme saisie immédiate de soi-même. Dans cette version flamboyante de la phénoménologie, le phénoménologue parvient, grâce à l'épochè, en vertu d'un sursaut dont il détient seul l'initiative - autrement dit : un acte absolu — à vaincre l'engourdissement du sens, lequel est considéré comme n'ayant été qu'un effet de surface, une illusion, un accident, en quelque sorte un malentendu, un (étrange) oubli du sujet transcendantal par lui-même ${ }^{1}$. Il s'agit de retrouver le sujet transcendantal éternel («l'esprit immortel»), mais l'étourderie passée d'un tel sujet devient dès lors une énigme. La thématique de la mort ou de l'assoupissement du sens exigerait plutôt que soit pris en compte un être capable de mourir et une phénoménologie par-delà l'opposition présence-absence. Et cela, me semble-t-il, peut être élaboré de façon très avancée à partir de l'étude husserlienne de l'imagination, à condition de surmonter l'hésitation entre une interprétation de l'imagination en termes d'acte ou comme mode d'être des choses.

\footnotetext{
Vergeistigung auferstehen, als Unterpfand einer großen en fernen Menschenzukunft: Denn der Geist allein ist unsterblich » (E. Husserl, Die Krisis der europäischen Wissenschaften und die transzendentale Phänomenologie. Eine Einleitung in die phänomenologische Philosophie (Husserliana VI), édité par W. Biemel, La Haye, Martinus Nijhoff, 1954, p. 348, trad. fr. G. Granel, La crise des sciences européennes et la phénoménologie transcendantale, Paris, Éditions Gallimard, 1976, p. 382-383). 1 La description, dans Erste Philosophie, d'une « autodissimulation (Selbstverhüllung) » du sujet transcendantal, d'un « me perdre dans le monde (Mich-verlieren in die Welt)», est ainsi particulièrement troublante et problématique. Cf. E. Husserl, Erste Philosophie (1923-1924), Zweiter Teil: Theorie der phänomenologischen Reduktion (Husserliana VIII), édité par R. Boehm, La Haye, Martinus Nijhoff, 1956, p. 77, trad. fr. A.L. Kelkel, Philosophie première, Paris, P.U.F., 1972, p. 107.
} 


\section{Actes ou modes d'être?}

\subsection{Phénoménologie des actes d'imagination}

Husserl refuse de fonder la différence entre perception et imagination sur une différence de contenu comme le faisaient les psychologistes. Ces derniers confondent objet visé et contenu sensible ${ }^{1}$, ils n'élaborent pas les notions d'acte intentionnel et de caractère d'acte (Akt-Charakter). C'est donc le contenu sensible qui porte à lui seul toute la différence entre perception et image. C'est alors de deux choses l'une. Soit l'on établit une différence de nature entre sensations et phantasmata (Phantasmen) (la hylé propre aux phantasiai) et l'on se rend alors incapable de comprendre qu'un même objet puisse être perçu ou imaginé ${ }^{2}$. Soit on considère que les phantasmata sont des sensations rémanentes mais affaiblies, ce qui rétablit une continuité entre perception et imagination mais remet en cause la fiabilité de la perception ${ }^{3}$. Tels sont les premiers arguments avancés par Husserl pour justifier l'idée qu'il faut impérativement penser l'imagination en termes d'actes, de modalité d'acte: il n'y a d'image que pour une certaine visée, un certain mode de l'appréhension qui accomplit une Verbildlichung, une mise en image ${ }^{4}$.

Ainsi dans la représentation-de-phantasia, le contenu hylétique doit être absolument homogène à celui des perceptions : il s'agit simplement d'appréhender des sensations actuelles sur le mode de la présentification d'un objet qui n'est pas là. La phantasia est «un acte de se présentifier (vergegenwärtigen) intérieurement ${ }^{5}$. Dans le $\S 22$ de la $6^{\mathrm{e}}$ Recherche logique, Husserl définit la représentation-de-phantasia ou présentification, comme appréhension analogisante de contenus hylétiques présents : au lieu d'être porteurs de l'être en personne de la chose elle-même, ils sont saisis comme ressemblants simplement à l'objet.

De même concernant la Bildbewusstsein : dans le cours de 1904-1905, Husserl distingue l'image physique de l'objet-image qu'il définit comme "l'image spirituelle (das geistige Bild) $)^{6}$. L'image physique est la chose présente ici et maintenant, la photographie, ce morceau de carton et quelques

1 Hua XXIII, § 3.

2 Hua XXIII, § 5, p. 10-11, tr. fr. p. 56 ; de même $\S 47$ : Husserl s'oppose pour cette même raison à l'idée générale d'une différence abyssale entre phantasma et sensation.

3 Hua XXIII, $\S 5$, p. 12, tr. fr. p. 58.

4 Hua XXIII. § 8, p. 16, tr. fr. p. 62.

5 Hua XXIII, p. 3 , tr. fr. p. 50.

6 Hua XXIII, p. 43 , tr. fr. p. 83. 
surfaces noires et blanches, grises ou colorées, la toile, les aplats de couleurs, etc. L'objet-image quant à lui est par exemple le petit personnage en noir et blanc qui apparaît dans l'image et me renvoie à mon ami Pierre. Le concept d'objet-image a d'abord pour fonction de souligner que le caractère d'image n'est pas une propriété qui serait déjà gravée, inscrite en soi dans les choses, mais un caractère constitué par un certain mode d'appréhension. Une simple chose physique ne peut en soi viser ou figurer autre chose et ce n'est pas parce qu'un objet ressemble à un autre qu'il est saisi comme son image : un jumeau n'est pas saisi normalement comme l'image de son jumeau ${ }^{1}$. Husserl insiste : l'image physique et l'objet-image ne sont absolument pas situés dans le même ordre de réalité. L'objet-image est ce qui flotte dans l'esprit, il est "l'image propre de l'imagination $»^{2}$. L'image physique est matérielle et présente en chair et en os ${ }^{3}$, tandis que l'objet-image «n'existe pas véritablement $»^{4}$, il est même défini comme un néant (ein Nichts) ${ }^{5}$. Ainsi Husserl aboutit à une conception phénoménologique de l'imagination comme acte : "L'image ne devient image que grâce à la faculté qu'a un moi doué de représentation d'utiliser le semblable comme représentant en image de ce qui lui est semblable » ${ }^{6}$. Et en effet, face à un tableau, conformément à cette approche en termes d'actes, il est bien vrai que je peux ou bien percevoir la toile et les couleurs ou bien les appréhender comme analogon représentant un paysage non actuellement perçu : il m'est possible de modifier mon acte.

On voit que cette théorie de l'imagination comme ancrée dans un acte d'appréhension et cette structure Auffassung-Inhalt, vont de pair avec une ontologie finalement classique et positiviste opposant présence et absence.

Le choix même du terme de Vergegenwärtigung témoigne de la primauté accordée par Husserl à la présence (Gegenwart) perceptive : la perception est l'expérience originaire, protoexpérience (Urerfahrung) ${ }^{7}$. La présentification serait donc une tentative pour re-produire une perception, tentative qui, du fait même de son caractère second et dérivé, serait vouée à n'accéder qu'à un ersatz de perception. Dans Ideen I Husserl la décrit également comme obscurcissement par rapport à l'intuition donatrice

1 Hua XXIII, p. 141, tr. fr. p. 164.

2 Hua XXIII, p. 19, tr. fr. p. 64.

3 Ibid. Et p. 506, tr. fr. p. 479.

4 «Wahrhaft existiert das Bildobjekt nicht», Hua XXIII, p.22, tr. fr. p. 66.

5 Hua XXIII, p. 110, tr. fr. p. 136.

6 Recherches logiques, $5^{\mathrm{e}}$ Recherche, Appendice aux $\S 11$ et 20.

7 Voir notamment Hua III p. 11 et 70, tr. fr., p. 15 et p. 126. 
originaire $^{1}$. Les Leçons sur la conscience intime du temps soulignent que la présentification, née d'un effort pour rappeler ou faire surgir ce qui n'est pas donné ici et maintenant, intègre un processus consistant à s'arracher à ce qui est présent et aller chercher du présent passé ou possible dans l'horizon des rétentions ou protentions ${ }^{2}$. Ce parcours défie en quelque sorte le cours du temps, lequel résiste et creuse, au sein même de la présentification, une distance irréductible: celle qu'il a fallu franchir et qu'il faut sans cesse franchir à nouveau pour rendre présent ce qui, décidément, ne l'est pas. La théorie de l'analogon est également corrélative de cette théorie de la reproduction : puisqu'on part d'une primauté de la présence, le seul moyen de rendre l'imaginaire quasi-présent, est de trouver un appui présent qui lui communiquera une présence par procuration.

Mais la compréhension de l'imagination comme étant essentiellement un acte soulève en fait plusieurs difficultés majeures, que Husserl affronte clairement à plusieurs reprises, ce qui le conduit me semble-t-il à avancer une théorie alternative beaucoup plus innovante.

\subsection{De l'acte au mode d'être flottant des choses dans les images et phantasiai}

La description centrée sur l'acte d'imagination se heurte à plusieurs problèmes et c'est précisément pour surmonter ces derniers qu'une conception alternative se fait jour.

Le premier problème majeur mis en valeur par Husserl est celui de l'affinité entre l'analogon et l'objet imaginé. Admettons que l'imagination, pour surmonter l'absence de son objet, consiste à s'appuyer sur un objet présent (analogon) pour viser un être absent, le présentifier au sens de produire

1 «Wir bevorzugten (...) die sinnlichen Anschauungen, insbesondere die von erscheinenden Realitäten, sowie die aus ihnen durch die Verdunkelung hervorgehenden und selbstverständlich mit ihnen durch Gattungsgemeinschaft vereinigten dunklen sinnlichen Vorstellungen » (Hua III, p. 284., tr fr., p. 392 : «Nous avons privilégié les intentions sensibles, en particulier celles qui portent sur des réalités qui apparaissent, ainsi que les représentations sensibles qui en procèdent par obscurcissement et qui de toute évidence leur sont unies par une communauté de genre »).

2 E. Husserl, Vorlesungen zur Phänomenologie des inneren Zeitbewußtseins (19051910), édité par M. Heidegger, Jahrbuch für Philosophie und phänomenologische Forschung, Bd. IX, repris par Max Niemeyer Verlag, Tübingen, 3. Auflage, 2000, p. 402-403 [36-37], trad. fr. H. Dussort, Leçons sur la conscience intime du temps, Paris, P.U.F., 1964, p. 60. 
une quasi-présence forcée et manquée. Une zone d'ombre demeure : comment comprendre l'origine de la relation entre l'analogon et le représenté ? Est-ce une relation instituée par le sujet, de manière complètement créative et arbitraire $^{1}$ ? L'on se demande alors de quelle façon l'analogon peut communiquer la moindre présence à l'imaginé et comment est possible une certaine présence intuitive de l'objet visé dans ce qui lui ressemble. D'autre part cette explication revient à nier le phénomène même de la ressemblance : je ne décide pas que le portrait ressemble à $\mathrm{x}$. Je ne peux projeter n'importe quelle imagination sur n'importe quel support. Il y a là des forces d'association qui m'affectent. On se tient ici, j'y reviendrai, au niveau des synthèses passives, une forme d'actes, certes, en termes husserliens, mais d'un genre problématique. Pour Husserl, précisément, il n'est pas question de considérer la relation entre analogon et objet représenté comme externe et absolument arbitraire. Je cite, dans l'appendice V du cours de 1904-1905 : « le semblable (...) a tendance à empiéter sur le semblable, à coïncider avec lui $»^{2}$. Il y a une «tendance à l'identification par coïncidence $»^{3}$, mais qui ne peut être qu'une "intention de coïncidence, mais à distance » ${ }^{4}$. Husserl avance donc ici l'idée d'une activité qui part des choses — idée que, je crois, il faut prendre au sérieux absolument — et, d'autre part, d'une ubiquité des choses qui déploient cette relation de ressemblance faisant d'elles plus qu'elles-mêmes et pourtant pas encore pleinement l'autre auquel elles

1 C'est la position que défend Sartre dans l'imaginaire, en mettant en avant les exemples adéquats de la rêverie projetant un visage dans les motifs du papier peint ou dans un nuage (L'imaginaire. Psychologie phénoménologique de l'imagination, Paris, Gallimard, 1940, « Folio essais », p. 76-78).

2 « Ähnliches (...) hat die Neigung, sich mit dem Ähnlichen zu überschieben, zu decken » (Hua XXIII, p. 142, tr. fr., p. 165).

3 « eine Tendenz auf deckende Identifizierung » (Hua XXIII, p. 143, tr. fr. p. 166). 4 «Die Beziehung auf das Sujet ist da, ist partiell in Deckungsintention, aber mit Abstand » (Hua XXIII p. 142, tr. fr. p. 165 : « Le rapport au sujet[-image] est là, est partiellement dans une intention de coïncidence, mais à distance »). Voir aussi Analysen zur passiven Synthesis. Aus Vorlesungs- und Forschungsmanuskripten (19181926), (Husserliana XI), édité par M. Fleischer, La Haye, Martinus Nijhoff, 1966, trad. fr. B. Bégout et J. Kessler avec la collaboration de N. Depraz et M. Richir, Grenoble, Ed. Jérôme Millon, 1998, p. 396 : « Si A éveille B “qui lui ressemble”, sur le mode de la résonance, alors $\mathrm{A}$ et $\mathrm{B}$ entrent dans l'unité de ressemblance, qui est une unité de consonance; bien qu'ils restent séparés ils fusionnent et entrent alors dans une sorte de recouvrement lointain » ("Wenn A das "ihm ähnliche” B weckt in der Weise der Resonanz, so tritt damit A und B in die Ähnlichkeitseinheit, und das ist eine Einheit der Konsonanz, das ist, sie "verschmelzen", sie treten, obschon gesondert bleibend, doch in eine Art Ferndeckung », Hua XI, p. 410). 
ressemblent. Cela nous conduit déjà vers l'idée d'une imagination anonyme précédant l'acte d'imagination : «anonyme » parce que c'est une motivation immémoriale, « imagination » parce que ce qui se déploie alors n'est ni pleine identité, ni radicale différence. L'on comprend aussi pourquoi, alors, ce qui est motivé est bien ici une imagination, pas une perception. De même Husserl reconnaît dans le paragraphe 44 d'Expérience et jugement que toute comparaison active suppose un premier lien entre les objets, lien qui ne doit pas être créé ex nihilo par la comparaison :

la comparaison comme activité, comme acte de contemplation relationnelle, comme va-et-vient actif du regard de saisie entre les membres de la relation, présuppose originairement une similitude ou une analogie "sensibles », un élément agissant dans la sensibilité avant toute saisie singulière et toute mise en relation. (...) Ce qui est donné dans le sensible exerce une stimulation, éveille l'intérêt inférieur qui se réalise dans la saisie singulière, et dans le parcours et le maintenir-ensemble des éléments rassemblés ${ }^{1}$.

Il y a donc, il doit y avoir, une analogie - un lien au-delà de l'alternative identité/différence - en amont de l'activité du sujet.

La deuxième difficulté à laquelle se heurte une théorie de l'imagination comme étant fondamentalement un acte est directement liée au problème précédent. La distinction chose-image d'un côté, objet-image, sujet-image de l'autre (autrement dit appréhension perceptive — appréhension imageante) demeure artificielle. Nous ne pouvons pas vraiment isoler une appréhension purement perceptive de la chose-image. Husserl le reconnaît de façon très claire à plusieurs reprises : «Je ne peux même absolument pas, mettons que je le veuille, repousser de côté l'apparition de l'objet-image et ne voir simplement que des lignes et des ombres sur le carton $»^{2}$. Par conséquent l'imagination part ici de la chose ; on doit admettre que, dans la présence perceptive de la photographie, du corps de l'acteur, du tableau, immanent à

1 « Das Vergleichen als eine Tätigkeit, als aktiv beziehendes Betrachten, aktives Hin- und Herlaufen des erfassenden Blickes zwischen den Bezogenen setzt ursprünglich voraus eine "sinnliche" Gleichheit oder Ähnlichkeit, ein in der Sinnlichkeit vor aller Einzelerfassung und Aufeinanderbeziehung Wirksames. (...) Das sinnlich Gegebene übt einen Reiz aus; es erwacht das Interesse niederster Stufe, Einzelerfassung und durchlaufende Zusammenhaltung zu üben » (E. Husserl, Erfahrung und Urteil. Untersuchungen zur Genealogie der Logik (1929-1939), rédigé et édité par L. Landgrebe, Hamburg, Glaassen und Gloverts, 1948, p. 224, trad. fr. D. Souche, Paris, P.U.F., 1970, p. 227).

2 Hua XXIII p. 488, tr. fr. p. 464. Husserl concédait déjà cela dans l'appendice II, p. 161. 
cette présence perceptive, se trouve un processus de référence à un autre absent, plus exactement quasi-présent. C'est l'objet perçu même, la présence perceptive en chair et en os qui se développe d'elle-même dans une certaine partie de son être comme venue à la quasi-présence d'un nouvel être.

Husserl aboutit à une thèse semblable lorsqu'il affronte une troisième difficulté : phénoménologiquement parlant la description des vécus de phantasia ou des consciences d'images en termes de re-présentation, de médiation, de retard de l'imagination par contraste avec une présence perceptive demeurant plus authentique, n'est pas juste. Elle ne l'est pas essentiellement, puisqu'elle vaut seulement pour les cas d'imagination mourante ou abstraite. Husserl le note dès 1898 :

Si nous sacrifions totalement à l'excitation d'une phantasia provoquée de façon vive, si, ainsi, nous nous mettons à vivre pleinement dans un monde phantasmé où les phantasiai, par leur enchaînement pertinent, leur exceptionnelle vivacité, leur plénitude individualisée, leur constance, leur indépendance ne le cèdent guère aux apparitions de la perception normale, là il n'y a rien à remarquer d'une fonction représentative des apparitions ${ }^{1}$.

La même description phénoménale vaut d'ailleurs pour les images physiques, ainsi, à propos de L'amour sacré et l'amour profane du Titien : " Je n'ai plus alors de sentiment d'impropriété quant au figuré, ce qui m'y intéresse c'est là, ce n'est pas indirectement représenté $»^{2}$. La quasi-présence est d'un type absolument inédit, ce que l'on n'arrive pas à comprendre si on veut en faire une re-production. Non seulement parce que Hamlet ne saurait être puisé dans mes rétentions ou dans mes protensions, il ne m'attend pas dans mes souvenirs ou mes anticipations de l'avenir, mais aussi plus essentiellement parce que, même si la présence d'Hamlet avait été effectivement vécue dans le passé, son voyage à travers l'espace et le temps jusqu'à la quasi-perception actuelle serait en soi une forme nouvelle et étonnante de ladite présence. La

1 «Wenn wir uns dem Reize lebendig erregter Phantasie ganz hingeben, uns in eine phantasierte Welt so recht einleben, wobei die Phantasien durch ihren sinnvollen Zusammenhang, durch die ausnehmende Lebendigkeit, individualisierte Fülle, durch Stetigkeit und Selbständigkeit den Erscheinungen der normalen Wahrnehmung kaum nachstehen; da ist von einer repräsentativen Funktion der Erscheinungen (...) nichts zu merken » (Hua XXIII, p. 119, tr. fr. p. 144). La même idée est exposée dans le $\S 8$ de l'Appendice 1 .

2 «Ein Gefühl der Uneigentlichkeit hinsichtlich des Dargestellten gar nicht aufkommt. Was mich dabei interessiert, das ist da, das ist nicht indirekt vorgestellt » (Hua XXIII, p. 155, tr. fr. p. 177; nous soulignons). 
présence distante imaginaire est inédite, elle vient compléter la notion générale de présence que l'expérience perceptive était en fait très loin d'avoir achevé d'explorer et d'élucider. C'est un nouveau mode de présence qui est donc en cause. Concernant la manière exacte de définir celui-ci, Husserl ne nous donne que des pistes. Une idée récurrente est que l'être imaginaire, quoique spatial et temporel, ne trouve pas d'ancrage dans le temps et l'espace objectifs. Il est impossible de situer le centaure dans cet espace actuel ou même dans un autre espace en un point qui pourrait être atteint par une voie déterminée débutant $\mathrm{ici}^{1}{ }^{1}:$ il se tient dans un temps et un espace parallèles. Sa présence est flottante : es schwebt mir vor ${ }^{2}$. Mais ces mondes parallèles font irruption dans l'actuel et perturbent la présence perceptive. Ils prennent donc la forme d'une nouvelle dimension de déphasage, de démultiplication et d'ubiquité au sein de notre monde. L'expérience imaginative n'est pas visée de la chose imaginaire sur fond de présence perceptive intacte, elle est bien plutôt la découverte d'une nouvelle dimension de la présence.

Ainsi Husserl insiste également à plusieurs reprises sur l'étrange concurrence non-frontale qui se noue entre l'imaginaire et le perçu : il n'y a pas contradiction entre le blanc perçu de cette tasse et le rouge imaginé de cette tasse, mais ils ne cohabitent pas pour autant de façon complètement pacifiée, le rouge peut aussi me captiver et m'entraîner dans un monde imaginaire de telle façon que le blanc passera complètement en arrière-plan, exactement comme lorsque je lis un roman captivant, ma situation ici dans ce fauteuil et les conditions triviales de ma propre vie s'éloignent infiniment : le retour à la réalité passe par un moment de malaise et, comme une oscillation, c'est une surimpression de deux mondes, un phénomène de basculement.

D'autre part ce basculement a bien lieu dans le monde et est une étrange potentialité des choses de ce monde, particulièrement exploitées dans les images physiques: dans une théorie de l'image qui n'est pas compatible avec celle de la représentation, Husserl parle de l'image physique, particulièrement du théâtre en ces termes : «Du point de vue du procédé, du rendu théâtral visé, de la composition nous disons: certaines choses s'avèrent propres à provoquer une double appréhension perceptive (doppelte perzep-

1 « Phantasmer [phantasieren] un A (...) veut dire laisser flotter (vorschweben lassen) cet objet, c'est-à-dire le laisser apparaître comme étant là lui-même (...). Mais bien sûr pas comme étant maintenant, comme étant là dans mon environnement de maintenant ! »(Hua XXIII, p. 174-175, tr. fr. p. 196).

2 Sur le concept de flottement, nous renvoyons à notre ouvrage La dimension imaginaire du réel dans la philosophie de Husserl, op. cit., p. 98-105.

81 
tive Auffassung) ${ }^{1}$. Le présent et la présence actuelle peuvent brusquement se dédoubler. Hamlet prend vie sur scène et nous l'imaginons non pas en utilisant le spectacle comme support, mais entraînés par le jeu de l'acteur: nous voyons et entendons Hamlet à même le corps, les paroles, les gestes de cet acteur. Hamlet n'est pas ailleurs, il n'est pas dans notre imagination, il n'est pas nulle part, il n'est pas un néant; ce sont toutes les personnes et les objets sur scène qui se métamorphosent. La seule différence avec des expériences purement perceptives est, affirme Husserl, que nous ne posons pas de croyance en leur existence, ni non plus en leur inexistence, en fait nous nous abstenons, l'expérience est «neutralisée $»^{2}$, non en vertu d'une décision arbitraire mais suivant une motivation portée par une certaine anomalie du spectacle $^{3}$.

Résumons : alors que la présence perceptive prétend me donner des objets clairs et en soi, la perception étant essentiellement liée à la croyance naturaliste, la présence flottante des images se livre essentiellement comme irruption d'un jeu sur des mondes parallèles, ou encore comme découverte d'un monde ontologiquement multidimensionnel.

Mais il faut encore accomplir un pas supplémentaire : en fait, ce flottement est une dimension ontologique de tout être. Tout en analysant la signification d'une telle thèse, je vais m'interroger dans la dernière partie de cet article sur le statut qu'il faut donner aux actes et à la passivité dans ce qui se profile comme une étrange imagination anonyme dans les choses mêmes.

\section{Imagination anonyme et actes fantômes}

Il est, me semble-t-il, extrêmement frappant de constater que, chez Husserl, le champ lexical de l'imaginaire (Phantom, Schema, Abschattungen, Schwebung...) s'invite régulièrement dans les textes où les actes imaginaires

1 Hua XXIII, p. 517, tr. fr. p. 488. Il s'agit d'une Perzeption, pas d'une Wahrnehmung, autrement dit, selon la terminologie husserlienne, l'objet est présent en chair et en os sans qu'advienne toutefois quelque prise de position, quelque croyance relative à cet objet (Hua XXIII, p. 466).

2 Hua XXIII, p. 515, tr. fr. p. 487.

3 Dans le cas des photographies ou des tableaux, les objets et personnages présentés, le plus souvent minuscules et de toute façon en deux dimensions, sont aisément perceptibles comme «anomaux » (Hua XXIII, p. 488, trad. fr. p. 464). Dans le cas du théâtre, la cérémonie du spectacle fait que par exemple je ne suis pas supposée monter sur scène pour utiliser les objets qui s'y trouvent, la table n'est plus une table « servant à quelque chose » (Hua XXIII, p. 518, trad. fr. p. 489). 
ne sont plus l'objet d'étude spécifiquement visé, mais où il est question de perception, du monde de la vie ou de la présence d'autrui par exemple. Je veux montrer dans cette dernière partie qu'il est légitime de parler d'une imagination anonyme à l'œuvre au niveau ontologique. J'expliquerai pourquoi, en choisissant de parler d'imagination anonyme ou d'actes fantômes encore au niveau ontologique, je maintiens une référence - qui ne peut que sembler problématique - à une faculté mentale d'imagination.

\subsection{Les choses flottantes du monde de la vie}

L'idée est clairement formulée dans la Krisis :

Les choses du monde ambiant intuitif se tiennent d'une façon générale, et pour toutes leurs propriétés, dans une certaine oscillation autour du type pur; leur identité avec elles-mêmes, leur être-égal-à-soi-même et leur durée temporaire dans une égalité, ne sont que des à-peu-près, de même que leur être-égal à d'autres choses ${ }^{1}$.

Dans la vie de l'expérience pré-scientifique (...) ce que nous nous approprions en tant que connaissance du même, (...) reste inévitablement quelque chose qui flotte dans l'à peu près (ein im Ungefähren in Schwebe Bleibendes) $)^{2}$.

Partons du concept d'Abschattung, d'esquisses : si la phénoménalité appartient à l'être - ce à quoi conduit en effet le rejet de la distinction kantienne entre phénomène et chose-en-soi - il me semble nécessaire d'intégrer le flux d'esquisses à l'être même des choses. Les textes d'Ideen I ne soutiennent pas tous cette thèse, et il importe en effet de distinguer la chose de ses esquisses. La chose ne coïncide avec aucune des esquisses en particulier. Elle est située dans l'espace objectif et saisie comme ne changeant pas alors

1 « Die Dinge der anschaulichen Umwelt stehen ja überhaupt und in allen ihren Eigenschaften im Schwanken des bloß Typischen; Ihre Identität mit sich selbst, ihr Sich-selbst-Gleischsein und in Gleichheit zeitweilig Dauern ist ein bloß ungefähres, ebenso wie ihr Gleichsein mit anderem » (E. Husserl, Die Krisis der europäischen Wissenschaften und die transzendentale Phänomenologie. Eine Einleitung in die phänomenologische Philosophie (Husserliana VI) (1936), édité par W. Biemel, 1954, La Haye, Martinus Nijhoff, p. 22, trad. fr. G. Granel, La crise des sciences européennes et la phénoménologie transcendantale, Paris, Gallimard, 1976, p. 29$30)$.

2 Hua VI, p. 357, tr. fr. p. 394. 
que les esquisses sont des vécus et changent sans cesse. Soit, mais ce sont les esquisses qui font signe les unes vers les autres et vers l'objectivation d'une chose apparaissant comme en soi à l'attitude naturelle. Husserl lui-même utilise des formules tout à fait frappantes : c'est bien la chose qui $s$ 'esquisse, «Das Ding sich “Abschattet” » ${ }^{1}$.

Toute réalité (...) se fait connaître peu à peu dans la perception qui la prend pour thème, tandis qu'elle se figure continûment (selbst da kontinuierlich darstellt) comme étant là dans son ipséité (...) se déployant dans ses notes, ses moments quidditifs singuliers ; ceux-ci, de leur côté, sont également présents à la conscience comme se figurant en eux-mêmes (sich selbst darstellende), mais comme ayant précisément le sens de moments dans lesquels la réalité en question s'indique en ce qu'elle est (sich zeigt als das, was es ist $)^{2}$.

La dimension de distance et d'inadéquation sont précisément l'élément constitutif essentiel d'une présence authentique, en chair et en os, de la chose même, dans sa transcendance.

Si la chose se tient dans cette oscillation entre esquisses, et dans ce flottement entre le flux des facettes apparentes et chose objectivée en horizon, alors il faut admettre qu'elle intègre un abîme d'indétermination et est également intrinsèquement riche d'avenir. Et, qui plus est, d'un avenir ouvert puisque, d'une esquisse à l'autre, ce n'est jamais une loi absolue, nécessaire, parfaitement claire qui nous est donnée et permettrait de définir à l'avance toutes les esquisses possibles au sein desquelles la chose pourra se profiler : la transcendance de la chose va de pair avec cette incomplétude de la loi d'enchaînement des esquisses ${ }^{3}$.

De même, dans Chose et Espace et Ideen II, Husserl étudie la manière dont, partant d'un flux d'esquisses ouvert, se forme la chose objectivée. Il

1 Ideen zu einer reinen Phänomenologie und phänomenologischen Philosophie, Erstes Buch : Allgemeine Einführung in die reine Phänomenologie (Husserliana III), (1913) édité par W. Biemel, La Haye, Martinus Nijhoff, 1950, p. 97, trad. fr. P. Ricœur, Idées directrices pour une phénoménologie transcendantale, Paris, Gallimard, 1950, § 42, p. 136 (et déjà p. 93, trad. fr. p. 132).

2 Erfahrung und Urteil, op. cit. p. 30, tr. fr, p. 40.

3 «Die Zukunft ist das Reich des Unbekannten, und als das ist es zunächst kein Reich des Ansich, kein Reich wahrer und in ihrer Wahrheit dem Ich vorgegebener Gegenständlichkeiten, sondern ein Reich der Unbestimmtheit » (Hua XI, p. 212, nous soulignons, trad. fr. De la synthèse passive, op. cit. § 46, p. 270 : « Le futur est le domaine de l'inconnu, et, comme tel, il n'est de prime abord ni le domaine de l'en $s o i$, ni le domaine des objectités vraies et prédonnées au moi dans leur vérité, mais un domaine de l'indétermination »). 
distingue une couche plus profonde de la chose, qu'il nomme son fantôme ou schéma, et la couche de l'être réel objectif. « Nous n'édifions ici que la chose dans sa couche inférieure (fantôme) ${ }^{1}$. De même dans Ideen I : «Toute apparence de chose enveloppe nécessairement en soi une couche que nous nommons le schéma de la chose $»^{2}$. Ricœur ajoute en note : "C'est ce que Ideen II appelle "le fantôme" ". Toute chose est d'abord un fantôme, c'est-àdire une certaine cohérence, ligne de cohérence qui se dessine, que le sujet le veuille ou non, dans une série d'esquisses, mais sans que l'on puisse encore bien délimiter les propriétés de la chose telles qu'elles se manifesteront à l'avenir. Certes on apprend à circonscrire de façon de plus en plus nette la chose qui reste la même alors que ses apparences changent, mais cela ne se fait que via une construction empirique qui demeure en fait toujours en chantier et sujette à révision. Ainsi, par exemple, la lumière change sans cesse, les perspectives aussi, mais je suppose que cette feuille de papier reste elle-même et que seule son apparence change. Pour que les modifications de l'apparence ne soient pas perçues comme altérations de la chose même, il faut que ces changements soient attribués à une transformation advenue dans l'un des objets du contexte auquel appartient cette chose ${ }^{3}$. Il faut qu'une relation de dépendance constante ait été observée entre les changements de l'éclairage ou de la position de mes yeux par exemple et les changements de l'apparence de l'objet (la feuille de papier). Une loi de relation causale peut alors être établie. L'objet enraciné dans le réel grâce aux relations causales devient discernable d'une pure illusion, il cesse d'être un fantôme ${ }^{4}$. Husserl parle de fantôme dès lors que l'on ne parvient pas à opérer une telle mise en ordre de l'expérience, de sorte qu'il est impossible de savoir si c'est la chose ou son apparence qui change : les deux registres coïncident. Le fantôme est un flux d'apparences changeantes et, du coup, imprévisibles, tandis que la chose réelle est supposée subsister sous le cours capricieux de ses apparences. Mais ce cadre formel de l'objectivation est dessiné peu à peu par

1 « Wir hier genau besehen nur das Ding nach seiner untersten Schicht (Phantom) aufheben » (Ding und Raum, Vorlesungen, 1907 (Husserliana XVI), édité par U. Claesges, La Haye, Martinus Nijhoff, 1973, p. 339, trad. fr. J.F. Lavigne, Paris, P.U.F., 1989, p. 392).

2 Ideen I, op. cit., p. 506 ( « jede Dingerscheinung notwendig in sich eine Schicht birgt, die wir das Dingschema nennen », Hua III, p. 370).

3 Ideen zu einer reinen Phänomenologie und phänomenologischen Philosophie, Zweites Buch : Phänomenologische Untersuchungen zur Konstitution (Husserliana IV) (1912-1917), édité par M. Biemel, La Haye, Martinus Nijhoff, 1952, p. 42, trad. fr. E. Escoubas, Paris, P.U.F., 1982, p. 72-73.

4 Ibid., p. 41, tr. fr. p.72. 
les comportements concordants des fantômes eux-mêmes et par leurs corrélations, ces lois se forment donc au cœur du flux des sensations et sont enrichies et éventuellement remodelées par lui. Il suffit par exemple qu'autrui me dise ne pas percevoir ces choses que je perçois pour que celles-ci soient rétrogradées en « hallucinations $»^{1}$.

Mais cette indétermination immémoriale et intrinsèque des choses ne saurait être une indétermination en soi. C'est la raison pour laquelle il me semble pertinent de parler d'imagination anonyme et d'actes fantômes.

\subsection{Les actes fantômes}

Dans le flux héraclitéen des sensations on trouve une formation de sens, des synthèses, qui constituent d'autre part des motivations reçues par les sujets, en d'autres termes des synthèses corrélatives d'une passivité du sujet. En effet si elles étaient pensées comme découlant originellement de l'acte d'un sujet transcendantal comme principe absolu, archè, et principe de jure accessible à une saisie intuitive immédiate de lui-même, alors elles ne fonderaient pas la présence d'un monde transcendant.

Husserl utilise le concept de synthèse passive pour désigner ces synthèses travaillant le flux sensible et qui relèvent de la pré-donnée, autrement dit se trouvent toujours déjà données à notre activité. Mais parler de synthèse passive permet en fait d'indiquer une étrange modalité de la passivité : le sujet ici n'est pas complètement passif et les synthèses sont une forme de subjectivité parente de la mienne. C'est pourquoi, me semble-t-il, les paradigmes ontologiques issus de l'étude du champ de l'imaginaire et de l'imagination peuvent être utilisés de manière pertinente et éclairante selon moi ici : on découvre ici un sujet transcendantal fantôme.

L'expression "synthèse passive » est indubitablement étrange : dans Dynamique de la manifestation, Renaud Barbaras en souligne les paradoxes et choisit de la révoquer. Une synthèse est par définition active, la dire passive revient à la rapporter au sujet qui la subit, mais dans ce cas l'on peut se demander pourquoi la synthèse est dite passive alors que c'est le sujet qui est passif.

Parler de synthèse passive, c'est dire trop ou trop peu : trop car si elle est vraiment passive, elle ne peut même pas être synthèse et la passivité du sujet correspond en réalité à une synthèse effectuée par le monde, à une forme

1 Ibid., p. 80, tr. fr. p. 121. 
d'activité de ce monde ; trop peu car si elle est vraiment synthèse, si elle s'enracine dans le sujet, elle ne peut être seulement passive et doit envelopper une dimension d'activité ${ }^{1}$.

Pourtant la notion husserlienne de synthèse passive cherche à penser une ambiguïté que, me semble-t-il, l'analyse de Barbaras exorcise. La transcendance du monde suppose que soit reconnue l'existence de synthèses qui ne soient pas dans une relation d'identité avec mes synthèses; mais ces synthèses sont, selon Husserl, familières en ce qu'elles sont en elles-mêmes indécises. Je citais plus haut quelques développements de Husserl à propos de la ressemblance : Husserl mettait alors en exergue le lien entre un sens naissant et ambigu et sa manifestation à nous sous la forme d'une «stimulation (Reiz) $»^{2}$. À plusieurs reprises dans De la synthèse passive, Husserl parle d'une sollicitation du sujet par ces synthèses. Elles constituent, dit-il, un « appel $(R u f){ }^{3}$, un appel à définir, à jouer avec les divers possibles offerts par cet à-peu-près, cette malléabilité ontologique des choses du monde de la vie. Husserl peut ainsi suggérer une personnalisation de l'arrière-plan passif : il m'appelle, «insiste »", il a ses propres visées, désirs, requêtes, ses entreprises engagées mais inachevées et encore hésitantes puisqu'il demande mon aide, laquelle est contingente et sera forcément particulière (mes reprises du monde de la vie peuvent ainsi être galiléennes et objectivantes ou phénoménologisantes). Il y a donc, dans les synthèses passives, du fait de l'indétermination au niveau du sensible, une distance à soi, une sorte de déphasage (retard/avance sur soi), qui se révèlent au sujet sous la forme d'un appel ayant « en soi » (et peut-être, du fait de cette distance, déjà pour soi) la forme interrogative, comme une liberté parlant à une liberté, une imagination parlant à une imagination, un art caché ${ }^{5}$ parlant à un sujet artiste.

Il est crucial que cette confuse familiarité des synthèses passives ne reste pas lettre morte, mais toute l'histoire de la sédimentation mise au jour par Husserl dans la Krisis montre que souvent et sans effort de notre part, le monde de la vie devient matière inerte, sens endormi, presque mort. Toute la phénoménologie husserlienne est fondée sur l'idée que je peux répondre à l'appel du monde de la vie, que je dois répondre, mais que, souvent, je ne

1 R. Barbaras, Dynamique de la manifestation, Paris, Vrin, 2013, p. 215.

2 Erfahrung und Urteil, p. 80, tr. fr. p. 89.

3 Hua XI, p. 7, trad. fr. De la synthèse passive, p. 98 : en chaque apparaissant résonne «l'appel: approche-toi plus près et toujours plus près, considère-moi et fixe-moi alors en changeant ta position, ton regard etc. ».

4 Erfahrung und Urteil, tr. fr. p. 90.

5 I. Kant, Kritik der reinen Vernunft, AK, III, 136, A141, B180.

87

Bull. anal. phén. XIII 2 (2017)

http://popups.ulg.ac.be/1782-2041/ @ 2017 ULg BAP 
réponds pas et laisse le sens se scléroser en croyant que le monde est un ensemble d'êtres en soi, massifs, non spirituels, aveugles et absurdes, en d'autres termes : de la matière morte.

C'est précisément parce que l'ouverture du sens donné à ma passivité doit essentiellement prendre la forme d'une liberté, d'un interlocuteur étrange et familier, que, aussi loin que l'on puisse remonter, nous trouvons toujours un arrière-plan qui est déjà vie et une subjectivité anonyme apparentée à ma subjectivité ${ }^{2}$. Je parle de sujets fantômes et d'actes fantômes parce que cette vie se trouve dans un rapport de corrélation essentielle avec la vie du sujet : son avenir dépendant de moi et ma pensée étant instituée par ces synthèses passives, ces dernières se décentrent en moi et je me décentre en elles. Les synthèses passives préfigurent mes actes et mes actes sont une réincarnation inattendue de ces synthèses, une reprise inopinée, libre, leur donnant un avenir qui n'était pas prédéterminé ; chacun des interlocuteurs la pré-donnée et le sujet qui s'en empare - devient ainsi, à travers ce dialogue un fantôme, un être au-delà de lui-même et de sa seule présence actuelle. Je me découvre comme précédée par un sujet qui est mon double et me parle, m'appelle, me sollicite. Me voici donc dédoublée. Corrélativement les synthèses passives existent déjà comme préfiguration des synthèses actives, en avance et en retard sur elles-mêmes, dédoublées elles aussi. Cette ubiquité ajoutée à l'oscillation de la vie à la mort et inversement sont précisément ce que je désigne par le terme de fantôme ${ }^{2}$.

Blanchot oubliait donc une troisième version de l'imaginaire : entre utiliser froidement la distanciation, l'irréalisation, à des fins d'idéalisation et de représentation, et sombrer dans une torpeur infinie, aspiré dans l'abîme du néant, il y a aussi, toujours et essentiellement, une imagination consistant à être appelé par l'être des choses et des personnes, à être entraîné par celui-ci dans un jeu de reprises et de création. C'est une corrélation essentielle entre un imaginaire anonyme qui naît en-deçà de mes actes et l'imagination active que cet imaginaire sollicite. Cette dimension imaginaire de toute chose est

1 D'une manière générale, le vivant est essentiellement un sens incarné et, indissociablement, ouvert, en chemin et créateur. La vie d'un organisme signifie en effet le déploiement d'une certaine cohérence (la vie n'est pas le chaos), d'un certain plan, d'un certain sens, mais ce, également à travers des transformations concrètes, des avatars, des maladies, des erreurs et quelques monstres qui s'enchaînent dans une série dont l'avenir apparaît comme inquiétant mais aussi riche de promesses.

2 Il y a encore beaucoup à dire sur ce concept de fantôme. Pour une analyse plus approfondie, je renvoie à la première partie de mon article « Esquisse d'une phénoménologie des fantômes » in Ostium, Revue Časopis des sciences humaines, L'invisible et le visible, vol. 2, 2015. 
également en évolution constante entre sédimentation et mort du sens l'indétermination et l'ouverture des choses de ce monde se trouvent voilées par les processus d'objectivation - et résurrection lorsque notre imagination s'en empare. Mais elle n'était jamais complètement morte et ses renaissances, dans les images et phantasiai, gardent la forme d'une quasi-présence, instable, évanescente, fragile. Au-delà de l'alternative entre présence et absence, vie et mort, c'est une prolifération des fantômes de chaque être et de chaque sujet qui est ainsi en cause.

\section{Bibliographie}

Barbaras Renaud, Dynamique de la manifestation, Paris, Vrin, 2013.

Baudrillard Jean, L'échange symbolique et la mort, Paris, Gallimard, 1976.

Blanchot Maurice, L'espace Littéraire, Paris, Gallimard, 1955.

Dufourcq Annabelle, «Esquisse d'une phénoménologie des fantômes " in Ostium, Revue Časopis des sciences humaines, L'invisible et le visible, vol. 2, 2015.

Dufourcq Annabelle, La dimension imaginaire du réel dans la philosophie de Husserl, Springer, Phaenomenologica, 2010.

Freedberg David, The Power of Images, The University of Chicago Press, 1989.

Husserl Edmund, Analysen zur passiven Synthesis. Aus Vorlesungs- und Forschungsmanuskripten (1918-1926), (Husserliana XI), édité par M. Fleischer, La Haye, Martinus Nijhoff, 1966, trad. fr. B. Bégout et J. Kessler avec la collaboration de N. Depraz et M. Richir, Grenoble, Ed. Jérôme Millon, 1998.

Husserl Edmund, Die Krisis der europäischen Wissenschaften und die transzendentale Phänomenologie. Eine Einleitung in die phänomenologische Philosophie (Husserliana VI), édité par W. Biemel, La Haye, Martinus Nijhoff, 1954, trad. fr. G. Granel, La crise des sciences européennes et la phénoménologie transcendantale, Paris, Éditions Gallimard, 1976.

Husserl Edmund, Die Krisis der europäischen Wissenschaften und die transzendentale Phänomenologie. Eine Einleitung in die phänomenologische Philosophie (Husserliana VI) (1936), édité par W. Biemel, 1954, La Haye, Martinus Nijhoff, trad. fr. G. Granel, La crise des sciences européennes et la phénoménologie transcendantale, Paris, Gallimard, 1976.

Husserl Edmund, Ding und Raum, Vorlesungen, 1907 (Husserliana XVI), édité par U. Claesges, La Haye, Martinus Nijhoff, 1973, trad. fr. J. F. Lavigne, Paris, P.U.F., 1989.

Husserl Edmund, Erfahrung und Urteil. Untersuchungen zur Genealogie der Logik (1929-1939), rédigé et édité par L. Landgrebe, Hamburg, Glaassen und Gloverts, 1948, trad. fr. D. Souche, Expérience et jugement, Paris, P.U.F., 1970.

Husserl Edmund, Erste Philosophie (1923-1924), Zweiter Teil : Theorie der phänomenologischen Reduktion (Husserliana VIII), édité par R. Boehm, La Haye, 
Martinus Nijhoff, 1956, trad. fr. A.L. Kelkel, Philosophie première, Paris, P.U.F., 1972.

Husserl Edmund, Ideen zu einer reinen Phänomenologie und phänomenologischen Philosophie, Erstes Buch : Allgemeine Einführung in die reine Phänomenologie (Husserliana III), édité par W. Biemel, La Haye, Martinus Nijhoff, 1950, trad. fr. P. Ricoeur, Idées directrices pour une phénoménologie transcendantale, Paris, Gallimard, 1950.

Husserl Edmund, Ideen zu einer reinen Phänomenologie und phänomenologischen Philosophie, Zweites Buch: Phänomenologische Untersuchungen zur Konstitution (Husserliana IV) (1912-1917), édité par M. Biemel, La Haye, Martinus Nijhoff, 1952, trad. fr. E. Escoubas, Paris, P.U.F., 1982.

Husserl Edmund, Logische Untersuchungen, Zweiter Teil. Untersuchungen zur Phänomenologie und Theorie der Erkenntnis, en deux tomes, La Haye, Martinus Nijhoff, 1984, trad. fr. H. Elie, A. L. Kelkel et R. Schérer, Recherches logiques, Paris, PUF, 1959-1963.

Husserl Edmund, Phantasie, Bildbewußtsein, Erinnerung. Zur Phänomenologie der anschaulichen Vergegenwärtigungen, Texte aus dem Nachlaß (1898-1925) (Husserliana XXIII), La Haye, Martinus Nijhoff, 1980, trad. fr. R. Kassis et J.F. Pestureau, Millon, Grenoble, 2002.

Husserl Edmund, Vorlesungen zur Phänomenologie des inneren Zeitbewußtseins (1905-1910), édité par M. Heidegger, Jahrbuch für Philosophie und phänomenologische Forschung, Bd. IX, repris par Max Niemeyer Verlag, Tübingen, 3. Auflage, 2000, trad. fr. H. Dussort, Leçons sur la conscience intime du temps, Paris, P.U.F., 1964

Kant Immanuel, Kritik der reinen Vernunft, AK, III, 136, A141, B180.

Sartre Jean-Paul, L'imaginaire. Psychologie phénoménologique de l'imagination, Paris, Gallimard, 1940. 